\title{
De la sostenibilidad a la sustentabilidad. Modelo de desarrollo sustentable para su implementación en políticas y proyectos
}

FECHA DE RECEPCIÓN: 10 de enero FECHA DE APROBACIÓN: 2 de febrero Pp. 40-55

Hernán Gustavo Cortés Mura* José Ismael Peña Reyes ${ }^{* *}$

A model of sustainable

development for the

implementation of policies and

projects

De la durabilité au développement durable : projets politiques pour une mise en place de modèles de développement durable

Da sustentabilidade para a sustentabilidade. Modelo de Desenvolvimento Sustentável para sua implementaçãu em politicas e projetos

"Ingeniero de Sistemas de la Universidad Nacional de Colombia. Magíster en Ingeniería Industrial; Docente auxiliar de la Facultad de Ingeniería de la Universidad Nacional de Colombia.

**Docente Facultad de Ingeniería de la Universidad Nacional de Colombia. Investigador en Gerencia, Gestión y Educación en Ingeniería y en Sistemas de Información. Doctor en Ciencias de Gestión - Sistemas de Información, Universidad de Grenoble, Francia. Master (Investigación) en Sistemas de Información y Organización. Universidad de Grenoble, Francia. Master Europeo MATIS Gerencia de Sistemas de Información, Universidad de Ginebra, Suiza. Especialización en Filosofía de la ciencia. Especialización en Pedagogía para el Desarrollo del Aprendizaje Autónomo. Ingeniero de sistemas de la Universidad Nacional de Colombia. 


\section{RESUMEN}

El desarrollo sustentable es un concepto que ha cobrado fuerza en los últimos años. Sin embargo, dadas sus características y sus múltiples aplicaciones, se ha prestado para ser interpretado y aplicado de maneras diversas. Este documento tiene un doble objetivo: presentar una revisión de la literatura para compartir algunos de los avances y discusiones en el campo del desarrollo sustentable; y discutir sobre la utilización y definición de los términos sustentabilidad y sostenibilidad, para finalmente proponer un modelo de desarrollo sustentable que permita a los tomadores de decisiones tener un punto de partida para trazar sus politicas de gestión.

\section{ABSTRACT}

Sustainable development has become a very important concept lately; however, due to its features and applications, it has been interpreted and applied in several ways. This paper aims at showing a bibliographic revision with some of the advances and discussions about sustainable development and to discuss about the use and definition of the term sustainability to finally propose a model of sustainable development that allows decision makers to have a starting point to design management policies.

\section{RESUMÉÉ}

Le développement durable est un concept ayant pris de l'ampleur ces dernières années. Cependant, en raison de ses caractéristiques et de ses multiples applications possibles, cette notion a été interprétée et appliquée de différentes manières. Cet article vise un triple objectif : présenter d'abord une revue de la littérature pour analyser certaines des avancées et discussions dans le domaine du développement durable; discuter ensuite de l'utilisation et de la définition de la durabilité et du développement durable et enfin proposer un modèle de développement durable qui offrira aux décideurs un point de départ pour élaborer leurs politiques de gestion.

\section{RESUMO}

O desenvolvimento sustentável é um conceito que recuperou força nos últimos anos. No entanto, dadas suas características e suas múltiples aplicações, se prestou para ser interpretado e aplicado de diversas maneiras. Este artigo tem um objetivo duplo: apresentar uma revisão da literatura para compartilhar alguns dos avanços e discussões no campo de desenvolvimento sustentável; e discutir sobre a utilização e definição dos termos de sustentabilidade e sustentabilidade, para finalmente propor um modelo de desenvolvimento sustentável que permita aos tomadores de decisões ter um ponto de partida para determinar suas políticas de gestão.

\section{Palabras clave}

\author{
Sustentabilidad \\ Sostenibilidad \\ Gestión
}

\section{Key words}

Sustainability

Sustainable

Management

Développement durable

Durabilité

Gestion

\section{Palavras-chave}

Sustentabilidade, Sustentabilidade

Gestão 


\section{INTRODUCCIÓN}

$\mathrm{E}$ I Mandato de Siete Generaciones (MSG) era el paradigma de la responsabilidad con las generaciones futuras de las tribus indoamericanas. Las reuniones del Consejo Tribal se iniciaban con la siguiente petición (oración) de orientación: "Oh Gran Espíritu, guíanos en las decisiones que vamos a tomar para que las consecuencias de la aplicación de nuestras definiciones sean positivas para nosotros y para nuestros hijos, ahora y por siete generaciones en el futuro" (Huisingh, 2006).

El Desarrollo Sustentable (DS) se ha convertido en un concepto aceptado a nivel mundial, para guiar las interacciones entre la naturaleza y la sociedad, con el fin de dominar los cambios locales y globales como cambio climático, inequidad social, pobreza, pérdida de biodiversidad, sobrepoblación y falta de recursos.

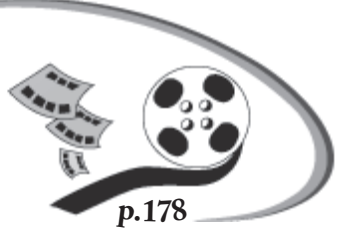

En tal sentido, se hace un llamado a un cambio de paradigma en todos los niveles, incluida la educación (Disterheft et al., 2013). No obstante, algunos estudiosos reconocen que por sus vagas características, el concepto de DS permite que coexistan varias definiciones e interpretaciones (Waas et al., 2011).
En la sociedad actual, las inquietudes ecológicas y las preocupaciones sociales son cada día más importantes. El DS ha tomado impulso para mantener su vigencia. Sin embargo, la penetración de nuestras prácticas insustentables sugiere que se están haciendo progresos insuficientes para cambiar de insustentable a vías sustentables (Huisingh, 2006). La sustentabilidad apunta hacia un futuro, hacia una solidaridad transgeneracional y un compromiso con las generaciones futuras (Leff, 2000). En 1987, la Comisión Brundtland de Naciones Unidas, en su informe "Nuestro futuro común" definió el desarrollo sustentable como: "aquel desarrollo que permite cubrir las necesidades presentes sin comprometer la habilidad de las generaciones futuras para cubrir sus necesidades" (Álvarez Etxeberria, 2009). Esta definición establece vínculos entre las dimensiones sociales, económicas y ambientales (Disterheft et al., 2013), sin embargo, es criticado por tener un enfoque antropocéntrico (Mebratu, 1998; Baker, 2005; Lozano, 2008; Waas et al., 2011).

En el crisol de la sustentabilidad se confrontan los tiempos de la degradación entrópica, los ciclos de la naturaleza y las crisis económicas, la innovación tecnológica y los cambios institucionales, con la construcción de nuevos paradigmas de conocimiento, comportamientos sociales y racionalidades productivas (Leff, 2000). 


\section{METODOLOGÍA}

E e realizó una revisión de la literatura compuesta de tres fases: búsqueda y detección, obtención y consulta, con el fin de analizar con detenimiento los datos bibliográficos centrados en los aspectos relacionados con el desarrollo sustentable y de integrarlos de una manera coherente y lógica.

En la búsqueda y detección se analizaron fuentes primarias que proporcionaron datos de primera mano y profundizaron en el tema. También, se consultaron fuentes secundarias que suministraron información esencial con el fin de obtener una visión global del tema. Finalmente, se consultaron fuentes terciarias, que hacian referencia a participaciones en eventos.
Una vez identificadas las fuentes, se procedió a localizarlas en las revistas académicas correspondientes y obtenerlas para su estudio detallado (obtención). Posteriormente, se realizó su lectura para extraer la información necesaria y relevante para la construcción de la revisión bibliográfica (consulta).

En seguida, se procedió a analizar la información para poder establecer una propuesta y sugerir la utilización e implementación de los términos sustentabilidad y desarrollo sustentable y de un modelo de desarrollo sustentable que permita a los tomadores de decisiones, tener un punto de partida para trazar sus políticas en el marco de este concepto.

\section{EL CONCEPTO DE DESARROLLO}

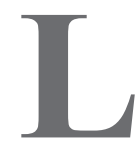
a noción de sustentabilidad tiene su origen en el ideal del desarrollo sustentable. Desarrollo no es sinónimo de crecimiento económico; este es sólo uno de los medios para lograr el primero. De modo tal, la implicación lógica del desarrollo sustentable es una economía material de crecimiento cero, combinada

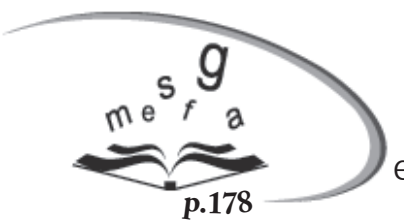

con una economía no material de crecimiento positivo. Mientras que el crecimiento demográfico y el crecimiento económico material, deberán estabilizarse con el tiempo. El crecimiento cultural, psicológico y espiritual no tiene límites físicos. Por lo tanto, el desarrollo sustentable es el que va del no desarrollo al desarrollo con crecimiento económico material, y luego, al desarrollo sin crecimiento económico material. En conclusión, este último es un proceso cualitativo de concretización de potencialidades que puede o no entrañar crecimiento económico (incremento cuantitativo de la riqueza) (Gallopín, 2003).

\subsection{Sostenibilidad y sustentabilidad}

En la literatura en español, se encuentra la utilización de los términos sostenible y sustentable; desarrollo sostenible y desarrollo sustentable; sostenibilidad y sustentabilidad. Sin embargo, quienes utilizan las expresiones se fundamentan en el término sustainability, como referente para sus publicaciones. Así por ejemplo, se pueden encontrar artículos científicos que traducen sustainability como sostenibilidad, y a su vez, otros artículos que traducen el término como sustentabilidad, incluso cuando la referencia citada es la misma. Méndez Chiriboga (2012), indica que tanto sostenibilidad como sustentabilidad no presentan mayor diferenciación con respecto a su aplicación al desarrollo, sino que su diferencia corresponde a su ubicación geográfica (lugar donde se utilice la expresión) o léxico, pero no modifica su objetivo principal: satisfacer las necesidades de la 
generación presente sin comprometer la satisfacción de las necesidades de las generaciones futuras.

Para establecer diferencias en los términos, se puede partir de criterios de uso conceptuales o lingüísticos. El diccionario de la Real Academia Española (RAE), define sustentable como: "que se puede sustentar o defender con razones"; y sostenible como "dicho de un proceso que puede mantenerse por sí mismo, como lo hace". Desde este punto de vista, Villamizar (s.f.) señala que el desarrollo sostenible es el término que se le da al equilibrio del manejo del Planeta en tres ámbitos: ambiental, social y económico. Teniendo en cuenta que ningún recurso renovable deberá utilizarse a un ritmo superior al de su generación, ningún contaminante deberá producirse a un ritmo superior al que pueda ser reciclado, neutralizado o absorbido por el medio ambiente, ningún recurso no renovable deberá aprovecharse a mayor velocidad de lo necesario para sustituirlo por uno renovable utilizado de manera sostenible.

Se refiere al desarrollo sustentable como un proceso integral que exige a los distintos actores de la sociedad compromisos y responsabilidades al aplicar mecanismos económicos, políticos, ambientales y sociales, así como en los patrones de consumo que determinan la calidad de vida. Requiere el manejo de recursos naturales, humanos, sociales, económicos y tecnológicos, con el fin de alcanzar una mejor calidad de vida para la población, y al mismo tiempo, velar porque los patrones de consumo actual no afecten el bienestar de las generaciones futuras (Villamizar, s.f.).

Por el contrario, Pujadas (2011) argumenta que sustainable development es un proceso de desarrollo económico, humano y medioambiental, que no depende de asistencia externa para mantenerse: un desarrollo sostenible en el tiempo. Mientras que no se ajusta el concepto sustentable al entenderlo como que se puede sustentar o defender con razones.

Ahora bien, desde el punto de vista lingüístico, Márquez Rodríguez citado por Villamizar (s.f.), descompone las palabras sostenible y sustentable mostrándolos como adjetivos verbales, porque derivan de sendos verbos: sostener y sustentar. Pertenecen al tipo de esos adjetivos que se forman mediante el agregado a la raíz del verbo del sufijo -able o -ible. Este denota la idea de posibilidad pasiva, es decir, capacidad o aptitud para recibir la acción del verbo. Sostenible es lo que es "capaz de sostener o de sostenerse", y sustentable aquello que es "capaz de sustentar o sustentarse". Márquez Rodríguez concluye que desde el sentido semántico, los términos son sinónimos.

En todo caso, los autores concuerdan con Villamizar (s.f.) en su conclusión, cuando a partir de la definición de los términos, indica que el desarrollo sostenible es aquel tipo de desarrollo que se da en una nación que puede mantener o sostener el equilibrio en la parte social, económica y ambiental; y desarrollo sustentable, es el tipo de desarrollo que genera una mejor calidad de vida, sin dejar que el consumismo afecte las generaciones futuras. Entonces, el desarrollo sostenible sólo se mantiene en la línea de estándares permitida, mientras que el sustentable, es aquel que crea o genera una mejor calidad de vida (Villamizar s.f.), de modo tal que este último implica todo lo que refiere el desarrollo sostenible pero lo lleva a su mantenimiento en el tiempo, sin afectar negativamente a las generaciones futuras. Por tal motivo, en el presente trabajo se utilizará el término sustentable y sus derivados, además de sugerir su utilización en cualquiera de sus campos de aplicación.

\subsection{Concepto de sustentabilidad}

Sustentabilidad y desarrollo sustentable han sido utilizados en la creciente literatura y en el debate sobre políticas con diferentes significados e interpretaciones (Pepper, 1998; Sathiendrakumar, 1996; citados por Martins et al., 2006). Hablar de sustentabilidad es, por lo tanto, hablar de desarrollo sustentable. La noción de desarrollo sustentable puede tener sus inicios en 1980, no obstante, la fecha que ha quedado registrada en la mayoría de los libros es el otoño de 1983, momento en el que la Organización de las Naciones Unidas crea la Comisión Mundial sobre el Medio Ambiente y el Desarrollo (CMMAD) (Viso, 2005). Sin embargo, el origen del concepto en sí se remonta 300 años en el trabajo sobre arboricultura sustentable de Hans Carl von Carlowitz (Saechsische, 2013) y TR Malthus 
(1766-1834) quien señaló los límites ambientales al crecimiento de la población (Mebratu, 1998). Por otra parte, para Gallopín (2003) el concepto de desarrollo sustentable es muy distinto del de sustentabilidad, en el sentido de que la palabra "desarrollo» apunta claramente a la idea de cambio, de cambio gradual y direccional. Lo que se sostiene, o debe hacerse sustentable, es el proceso de mejoramiento de la condición humana (o mejor, del sistema socio-ecológico en el que participan los seres humanos), proceso que no necesariamente requiere del crecimiento indefinido del consumo de energía y materiales.

Para avanzar hacia el desarrollo sustentable se necesita:

- Eliminar las rigideces y obstáculos acumulados.

- Identificar y proteger la base de conocimientos y experiencias acumulados que son importantes como los cimientos para avanzar.

- Sostener las bases sociales y naturales de adaptación y renovación, e identificar y acrecentar la capacidad necesaria de renovación que se ha perdido.

- Estimular la innovación, la experimentación y la creatividad social.

Se puede entender la sustentabilidad como un paradigma para pensar en un futuro en el que las consideraciones ambientales, sociales y económicas se balanceen en la búsqueda del desarrollo y una mejor calidad de vida (Mckeown et al., 2002). Finalmente, se enfatiza en que el concepto de desarrollo sustentable debe tener unas bases éticas, como pueden ser la justicia intergeneracional, la equidad intrageneracional o la preocupación ecocéntrica de preservación de la diversidad biológica (Biofilia) (Gallopín, 2003).

La sustentabilidad es un proceso de manejo adaptativo y pensamiento sistémico, que requiere creatividad, flexibilidad y reflexión crítica (Tilbury 2003, IUCN 2004, citados por Tilbury, 2004). El DS y las exigencias que esto pone a particulares y profesionales, está en constante evolución (Hanning et al., 2012). Despejando al desarrollo sustentable de su connotación exclusivamente ecológica y medioambiental (Santos Rego, 2009), Gro Harlem Brundtland fija y populariza la expresión tal como se mencionó anteriormente. De tal manera, la búsqueda de la sustentabilidad y del desarrollo sustentable exige integrar factores económicos, sociales, culturales, políticos y ecológicos (CNUMAD, 1992; Gallopín y otros, 2001; Kates y otros, 2001; citados por Gallopín, 2003). La idea de desarrollo sustentable está también insaturada: desarrollo sustentable, ¿De qué? ¿De la economía ¿Del medio ambiente? ¿De las capacidades humanas? ¿De un plan general de ordenación urbana? También, la industria del armamento podría desarrollarse sustentablemente y eso no la convertiría inmediatamente en deseable.

Foster, es recogido por Vare and Scott, (2007), cuando afirma que el desarrollo sustentable es un proceso de hacer que el futuro emergente sea ecológicamente sano y humanamente habitable tal como surja, a través del aprendizaje continuo donde la especie humana es la más dotada. Es un proceso de aprendizaje social de mejoramiento de la condición humana. Y es un proceso que se puede continuar indefinidamente sin socavarse así mismo.

El DS abarca tres categorías: medioambiental (medio ambiente, herramientas de evaluación, recursos, tecnologías verdes), social (valores e impactos sociales) y económicas (aspectos económicos, la gestión, las partes interesadas, la política y las políticas) (Hanning et al., 2012). Martins et al. (2006), presenta este modelo general aceptado para la sustentabilidad (Figura 1).Por otra parte, para Viso (2005), la sustentabilidad se puede tratar desde cuatro perspectivas: medioambiental, económica, ética y gobernanza sustentable (Figura 2). 
Figura 1. Modelo para la sustentabilidad.

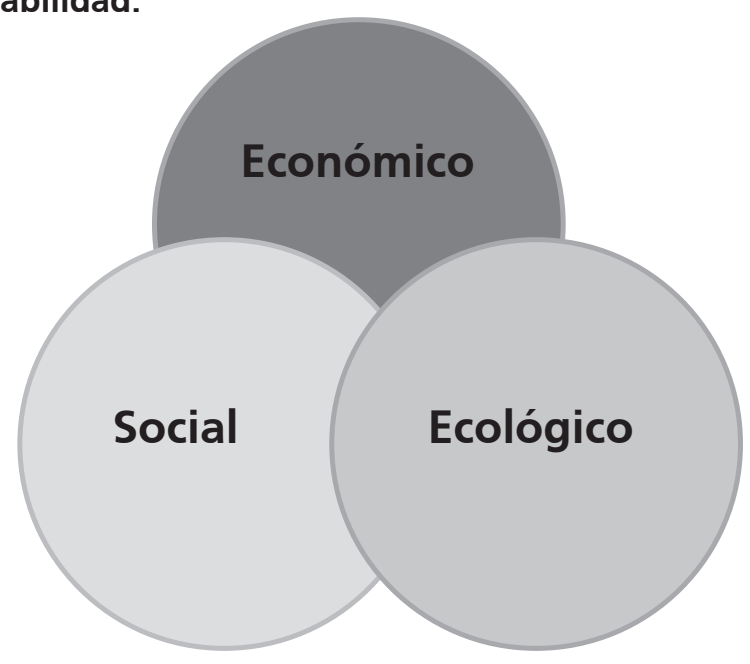

Fuente. Elaboración propia , a partir de Martins et al, (2006).

Figura 2. Modelo de sustentabilidad

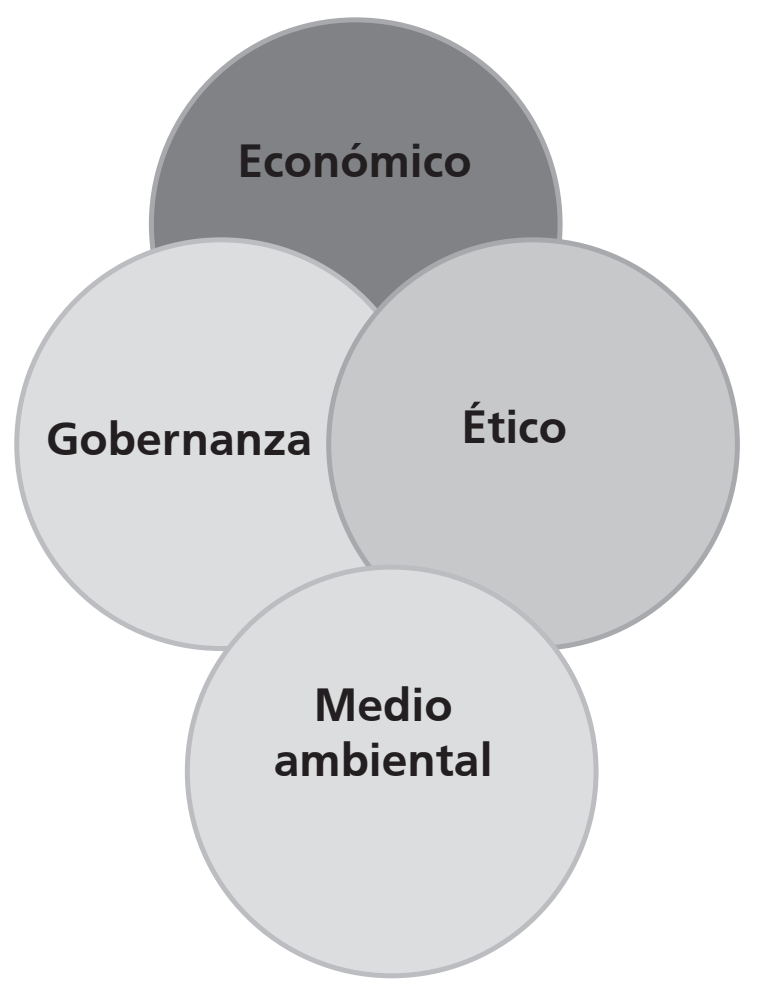

Fuente.Elaboración propia, a partir de la propuesta de Viso, (2005)

En los últimos años el modelo ha añadido una cuarta y quinta dimensión: institucional y cultural (Waas et al., 2011). Un modelo ideal compara, por ejemplo, los principios normativos, la gobernanza, la tecnología, la integración de políticas y herramientas y la filosofía subyacente de cada modelo; permite, según
Baker (2005), moverse desde una visión del mundo antropocéntrica a una ecocéntrica, con conceptos de DS más fuertes. De tal manera se puede considerar el DS como un proceso de aprendizaje social (Mulder et al., 2012). 
Como la ética y la gobernanza, así como las dimensiones institucional y cultural pueden enmarcarse dentro del componente social descrito anteriormente, es suficiente contar con las categorías medioambiental, ética y económica para trabajar correctamente el DS y como complemento contar con las demás en forma de modalidades de las principales.

Empresarios y ejecutivos están impulsando el cambio hacia la sustentabilidad en sus firmas, implementando por ejemplo: reorientación de estructuras organizativas y de estrategias, nuevas políticas de comunicación y relaciones públicas con agentes sociales implicados, cambios en las prácticas de gestión, introducción del reporte triple (económico, social y medioambiental), y a su vez, se multiplican los consultores especializados en la materia (Alvarez Etxeberria, 2009). La civilización parece estar al borde de una tercera revolución, la revolución de la sustentabilidad, las dos primeras son la industrial y la agrícola (Counce et al. 2003, citado por Glavic, 2006). La sustentabilidad replantea la pregunta por el Ser y el tiempo desde el cuestionamiento sobre la racionalidad económica, sobre la ontología y la epistemología que fundan una comprensión del mundo que ha derivado en formas de dominación de la naturaleza (Leff, 2000).

Según Gallopín (2003), la sustentabilidad puede definirse en términos elementales como $V(\mathrm{O} t+1) \leq \mathrm{V}$ $(\mathrm{Ot})$, Donde $V$ es la función de valuación de las salidas o productos del sistema (esto es: un sistema es sustentable cuando el valor neto del producto obtenido, no necesariamente en términos económicos, no disminuye en el tiempo). Toda asignación de valor contiene un componente subjetivo, por lo que la especificación de la función de $V$ y sus variables de salida, pueden variar ampliamente y reflejar la gama de percepciones y puntos de vista con respecto de las relaciones entre la naturaleza y la sociedad. Para algunos, $O$ no es más que el acervo total de capital, y $V$ una medida monetaria de ese capital. Para otros, $V$ es algún tipo de función agregada de bienestar, y $O$ puede estar diferenciado en capital natural, manufacturado y social. $\vee$ puede ser una función de valuación que incluya algunas prioridades éticas para la conservación de todas las especies vivas y estar expresada en unidades no monetarias (Gallopín, 2003).
En la comunicación sobre responsabilidad social corporativa de marzo de 2006, la CMMAD se ha fijado el objetivo de trabajar con todos los grupos de presión relevantes para hacer de Europa un polo de la excelencia sobre Responsabilidad Social Empresarial (RSE). Convencidos de que el potencial de la RSE puede colaborar en el desarrollo sustentable, el crecimiento europeo y la estrategia de empleo, la CMMAD sugiere la RSE como práctica para mejorar el uso de los recursos naturales para potenciar el rendimiento de la innovación y reducir la pobreza, así mismo, aumentar el respeto hacia los derechos humanos (UE, 2006; citada por Álvarez Etxeberria, 2009), teniendo en cuenta que los problemas ambientales están íntimamente relacionados con cuestiones sociales y políticas (Mulder, 2004). Las ciudades pueden gobernarse de manera sustentable y enfrentar la emergencia de problemas como la inseguridad, la escasez de recursos hídricos y energéticos y la exclusión social, y hacer posible la coexistencia de la diversidad cultural y social, porque lo que está en riesgo es la evolución biológica de más de 4.000 millones de años. La sustentabilidad pasa por la integración cognitiva de los saberes, la información y los conocimientos alcanzados por las ciencias (Hernandez García et al., 2012).

En el plano socio-comunitario, los proyectos de educación intercultural deben diseñarse y comprenderse en línea de relación directa con los proyectos de sustentabilidad (Santos Rego, 2009). Es necesario, por ello, impulsar el compromiso social y la participación, establecer compromisos de acción en los centros educativos y de trabajo, en las ciudades, en las propias viviendas para poner en práctica algunas de las medidas y realizar el seguimiento de los resultados obtenidos. Estas acciones, debidamente evaluadas, se convierten en el mejor procedimiento para una comprensión profunda de los retos y en un impulso para nuevos compromisos (Vilches \& Pérez, 2012).

La celebración de la Cumbre de la Tierra en Río de Janeiro en 1992, supuso un punto de inflexión en el reconocimiento del papel de la educación, y en particular, de la educación universitaria, para contribuir a la solución de los problemas globales que afectan además de avanzar hacia un desarrollo sustentable 
(Agenda 21, capítulo 36, 1992). En dicha Cumbre se hizo un llamamiento para que los educadores de todos los niveles y disciplinas contribuyeran a que la ciudadanía adquiriera una visión adecuada de los problemas y desafíos que afectan a la humanidad y pudieran participar así en la necesaria toma de decisiones fundamentadas (Vilches y Gil, 2003; citado por Vilches \& Pérez, 2012).

Se adoptó la sustentabilidad como principio fundamental para apoyar el desarrollo de la humanidad en todos los niveles. Se reafirmó la importancia de la educación para la sustentabilidad y la necesidad de considerar los aspectos sociales, económicos y políticos del desarrollo sustentable (Martins et al. 2006). En un entorno de crisis como el actual, se recuerda más las funciones sustanciales de la universidad, la formación y la investigación, como parte importante de las soluciones a los problemas que enfrenta nuestra sociedad, entre ellos, el de su insustentabilidad (Alba Hidalgo et al., 2012).

Es necesaria entonces la sustentabilidad del sistema socioecológico total. A largo plazo, la única opción que tiene sentido, es procurar alcanzar la sustentabilidad del sistema socioecológico completo. Las razones que justifican tener en cuenta el sistema como un todo, es la existencia de importantes vinculaciones entre sociedad y naturaleza. Se entiende por sistema socioecológico (Gallopín y otros, 1989; citados por Gallopín, 2003), un sistema formado por un componente (subsistema) societal (o humano), en interacción con un componente ecológico (o biofísico). La Carta de la Tierra es una declaración de principios fundamentales para la construcción de una sociedad global justa, sustentable y pacífica en el siglo XXI. Los principios fundamentales de la Carta de la Tierra son (Huisingh, 2006):

- Respeto y cuidado de la comunidad de vida.

- Integridad ecológica.

- Justicia social y económica.

- Democracia, no violencia y paz.

Para celebrar el aniversario 20 de la Declaración de Tbilisi sobre Educación Ambiental (1977), la Conferencia de Tesalónica de Educación Ambiental (1997) presentó una declaración que sería adoptada por todos gobiernos participantes. Esta declaración afirma que el avance en la Educación Superior (ES), sigue sin explotar y aún queda un gran trabajo por hacer, (Martins et al. 2006). Algunos de los indicadores de sustentabilidad que han sido propuestos consisten en: reducir los impactos que tiene la actividad humana sobre el medio ambiente, en especial, las tasas de utilización de los recursos renovables y no renovables; no superar la capacidad de carga de los recursos naturales y ecosistemas; integrar los objetivos a largo plazo económicos, sociales y ambientales; y preservar la diversidad biológica, cultural y económica (Bergh y Jeroen, 1996; citados por Gallopín, 2003). En su estudio, Bergh y Jeroen (1996) citados por (Gallopín, 2003), muestran diferentes puntos de vista teóricos sobre el desarrollo sustentable, del cual se resalta la teoría "ético-utópica", que caracteriza el desarrollo sustentable como "Nuevos sistemas individuales de valor (respeto por la naturaleza y las generaciones futuras, satisfacción de las necesidades básicas) y nuevos objetivos sociales (estado estacionario); atención equilibrada a la eficiencia, distribución y escala; fomento de actividades en pequeña escala y control de los efectos secundarios (lo pequeño es hermoso); política de largo plazo basada en valores cambiantes y estimulante del comportamiento ciudadano (altruista) en contraposición al comportamiento individualista (egoísta)" (Bergh y Jeroen (1996) citados por Gallopín, 2003).

Es imprescindible impulsar un desarrollo endógeno desde abajo y la gestión participativa de proyectos integrales, mediante fórmulas innovadoras y creativas de asociación local, capaces de hacer converger capacidades y valores del desarrollo sustentable, donde el desarrollo humano de la población y sus condiciones de vida tienen prioridad real, porque estas dimensiones son las que verdaderamente potencian un desarrollo sustentable y duradero. Transferir esta filosofía de la sustentabilidad al desarrollo local sustentable, requiere de un mayor control democrático, transparencia (Herranz, 2007; citado por Seller, 2012), innovación (Marcuello y Sanz, 2008; citado por Seller, 2012), y de una participación real y de impacto perceptible en las decisiones del medio local por parte de los ciudadanos (Seller, 2012). La Organización de Estados Iberoamericanos para la Educación, la Ciencia y la Cultura (OEI) dedica dos 
documentos que sintetizan las acciones concretas que se pueden realizar como ciudadanos (Vilches \& Pérez, 2012), algunas de ellas son:

- ¿Cómo podemos contribuir cada ciudadana y ciudadano a construir un futuro sustentable?

- Estudio de la problemática de la sustentabilidad (estudio de la literatura básica, publicaciones).

- Incorporación de la sustentabilidad en la enseñanza en currículos de los diferentes niveles, evaluación de cambios de actitud y comportamiento, elaboración y evaluación de materiales, etc.

- Contribución a la incorporación de la sustentabilidad en las diferentes actividades de la organización: crear y participar en comisiones de centro para establecer compromisos de consumo responsable, separación y tratamiento de recursos, movilidad sustentable, realizar seguimiento, etc.

- Participación en tareas de investigación e innovación para la sustentabilidad: impulsar proyectos de investigación en el campo de la sustentabilidad, el intercambio entre equipos, participar en investigaciones en torno a los cambios de actitudes y comportamientos sustentables, impulsar las publicaciones y secciones específicas en las revistas en torno a la sustentabilidad, publicar libros, capítulos de libro sobre la problemática, etc.

- Apoyo a la década de la educación por un futuro sustentable y a otras convocatorias de organismos internacionales que contribuyan al logro de la sustentabilidad (Cumbre de Río +20, Año Internacional de la Energía sustentable para todos, etc.)

Cada día aparecen nuevos ejemplos que muestran que el desarrollo económico, el desarrollo social y la protección del medio ambiente, resultan a menudo, metas mutuamente excluyentes. Lo deseable es encontrar un auténtico equilibrio estable que deje fuera las actividades incompatibles entre sí y por tanto, simultáneamente insustentables (Viso, 2005). Para mantener cada uno de los mecanismos necesarios para impulsar la sustentabilidad, las organizaciones, gobiernos, autoridades fiscales, reguladores de mercados y otros agentes, necesitan contar con información útil y creíble (Álvarez Etxeberria, 2009). Los informes sociales y medioambientales separados representan un paso adicional en la atención a los grupos de interés (Gray et al., 1996; citado por Álvarez Etxeberria, 2009).

En el marco de este tipo de documentos, existe una amplia gama de opciones: informes medioambientales, informes sociales, informes de RSC, etc. Sin embargo, la acepción más extendida es la de memoria de sustentabilidad, posiblemente debido a la denominación de mayor referente internacional, la Global Reporting Iniciative (Moneva, 2007, citado por Álvarez Etxeberria, 2009). De esta manera, la utilidad social del conocimiento consiste en la producción de cambios en los hábitos de vida y en la adopción de transiciones necesarias para elegir opciones de futuro viables (Hernández García et al., 2012).

En el pensamiento reciente sobre el desarrollo sustentable, el aprendizaje y el cambio, Scott y Gough (citados por Vare \& Scott, 2007) identificaron tres tipos de enfoque:

- Los enfoques que asumen que los problemas que enfrenta la humanidad son esencialmente ambientales, se pueden entender a través de la ciencia y resolverse por acciones y tecnologías medioambientales y/o sociales adecuadas. Supone que el aprendizaje lleva al cambio una vez que se establecen los hechos y se dice a la gente lo que son.

- Enfoques que asumen que los problemas fundamentales son sociales y/o políticos, y que estos problemas producen síntomas ambientales. Estos problemas fundamentales pueden ser entendidos por medio de cualquier cosa, desde el análisis de las ciencias sociales hasta una apelación al conocimiento indígena.

La solución, en cualquier caso, es lograr el cambio social, donde el aprendizaje sea una herramienta para facilitar la elección entre alternativas futuras que se pueden especificar con base en lo que se conoce 
en la actualidad. En el tipo de enfoques uno y dos, los estudiantes están ahí, en términos generales, para aprender a valorar lo que otros les dicen que es importante. Ambos enfoques tienen una larga historia y son atractivos para los grupos que defienden y presionan por un cambio hacia la sustentabilidad.

Un tercer enfoque asume que lo que es y puede ser conocido en el presente, no es suficiente; estados finales deseados no pueden ser especificados. Lo que significa que todo aprendizaje debe ser abierto. Este enfoque es esencial si la incertidumbre y complejidad inherentes a la forma en que se vive actualmente son llevadas a un aprendizaje social reflexivo acerca de cómo se podría vivir en el futuro. Para lograr alcanzar la sustentabilidad, es necesario entender su funcionamiento y hacerla parte del cotidiano de quienes pueden enfrentar la crisis ambiental y social, e implementar acciones coordinadas y constituyentes de una nueva vía para la humanidad. Ya sea que el desarrollo sustentable sea considerado como nuestro mayor reto (Annan, 2005 citado por Vare, Scott, 2007) o una letanía subversiva (Lomborg, 2001, citado por Vare, Scott, 2007). De tal modo, parece ser aceptable perseguir el cambio tecnológico para contribuir al desarrollo sustentable.

Sin embargo, el enfoque tecnocrático tradicional de arriba hacia abajo, no va de la mano con el desafío del desarrollo sustentable. La democratización de la toma de decisiones tecnológicas y el aumento de la participación de los grupos de interés, son importantes para evitar los errores del pasado (Mulder, 2004), la educación superior, se considera fundamental para el progreso en DS (Niu et al., 2010).

\section{RESULTADOS}

D espués de realizar el análisis bibliográfico, se propone un modelo que recoge diferentes elementos de los recogidos en el presente artículo. Se sugiere como un esquema que ubica los elementos claves de la sustentabilidad para ser tenidos en cuenta en el desarrollo de proyecto o políticas que quieran enmarcarse en el concepto de desarrollo sustentable.

\subsection{Utilización de términos}

Se sugiere que se apliquen los términos sustentabilidad, sustentable y desarrollo sustentable en la totalidad de sus dimensiones teniendo en cuenta la diferenciación con los términos sostenibilidad, sostenible y desarrollo sostenible por cuanto su aplicabilidad, y sobre todo, por su intención de hacer durable en el tiempo ese equilibrio entre sus componentes y así poder considerar la gestión del proyecto o la política desde un verdadero enfoque sustentable.

\subsection{El modelo}

Se involucran elementos recogidos de la literatura que han sido desarrollados en el presente artículo. En ese sentido, dichos elementos, que en principio toman como base alguna construcción encontrada en la literatura, han sido ajustados para encajar en el modelo de desarrollo sustentable propuesto. El modelo, es propuesto con el fin de aportar en la discusión académica en cuanto a la utilización de los términos, y pretende plasmar de manera concreta las dimensiones y modalidades que se deben tener en cuenta para involucrar el DS en la ejecución de un proyecto, planteamiento de políticas o procesos de gestión mismos de una organización.

Es necesario entender entonces el desarrollo sustentable, como aquel que va del no-desarrollo al desarrollo con crecimiento económico material, y luego, al desarrollo sin crecimiento económico material. Implica el manejo de recursos naturales, humanos, sociales, 
económicos y tecnológicos, con el fin de alcanzar una mejor calidad de vida para la población, y al mismo tiempo, velar porque los patrones de consumo actual no afecten el bienestar de las generaciones futuras. Es buscar el equilibrio entre las dimensión social, económica y medioambiental (Figura 3), teniendo en cuenta la variable tiempo en su concepción, de modo tal que no se comprometa la posibilidad de satisfacción de las necesidades de las futuras generaciones (Figura 4).
Cada una de las dimensiones contiene modalidades que son las que van a reflejar las políticas o a concretar el desarrollo sustentable en un proyecto. Sugiere determinar qué temas se van a trabajar, dependiendo de las condiciones objetivas de la organización y la posibilidad de incorporar las modalidades en cada una de las dimensiones, de acuerdo con la naturaleza y fines de la organización (Figura 5).

Figura 3. Equilibrio entre las dimensiones del desarrollo sustentable

Fuente. Elaboración propia de los autores, (s.f.).

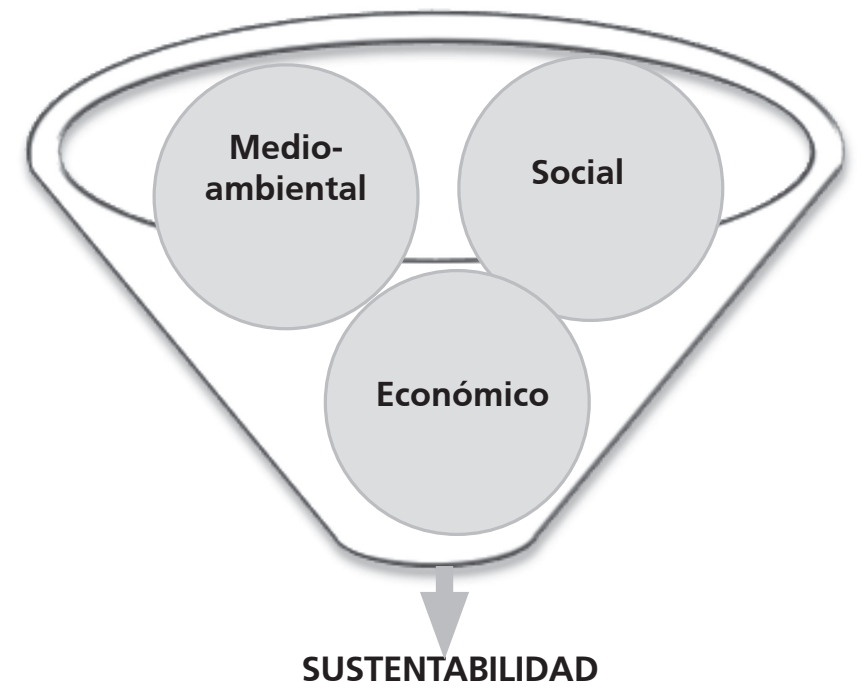

Figura 4. Desarrollo sustentable permanente en el tiempo.

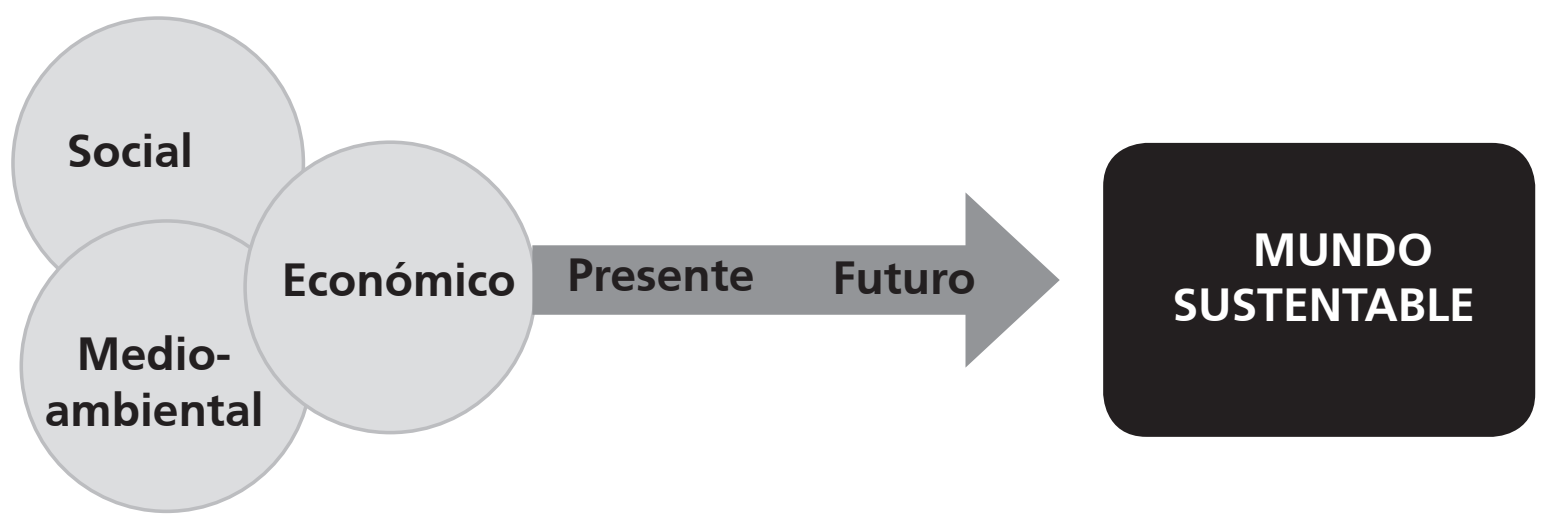

Fuente. Elaboración propia de los autores, (s.f.). 
Figura 5. Modalidades y dimensiones del desarrollo sustentable
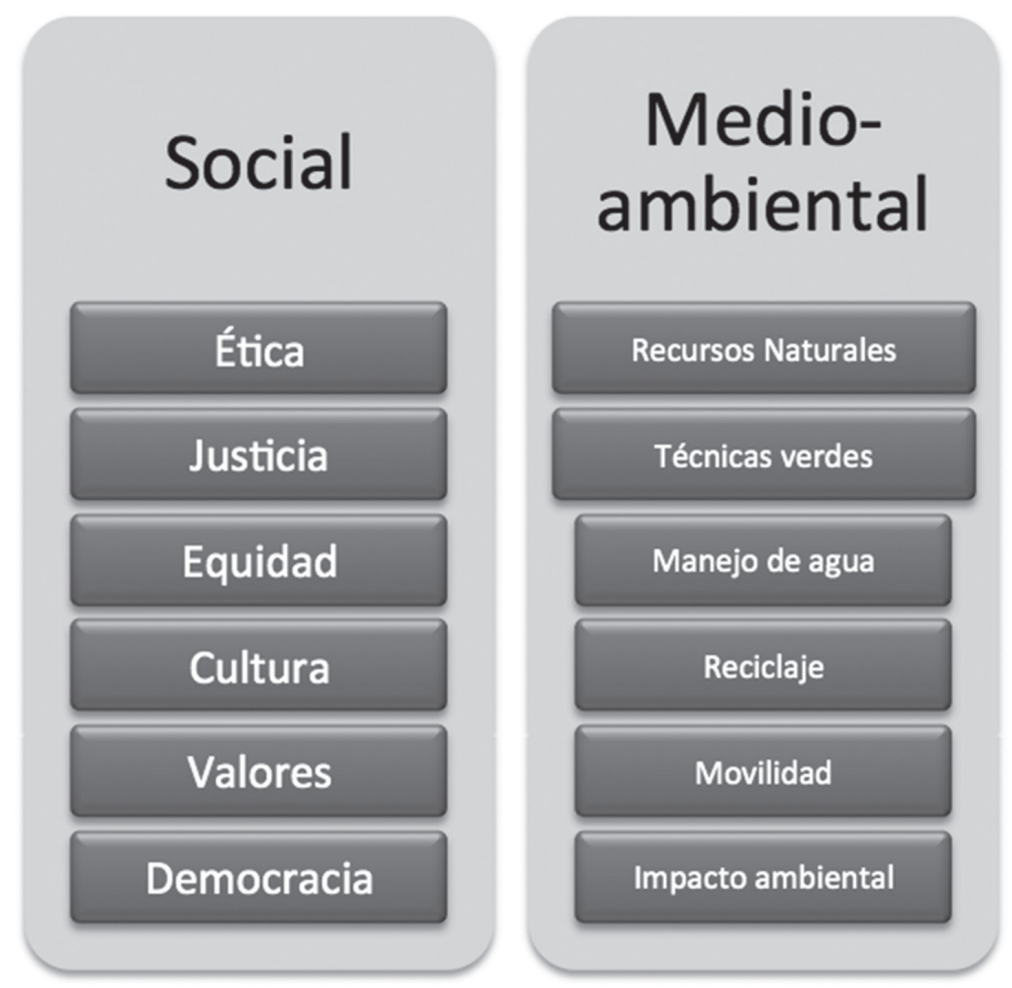

\section{Económico}

Economía material con crecimiento finito

Economía no material

con crecimiento positivo, no finito

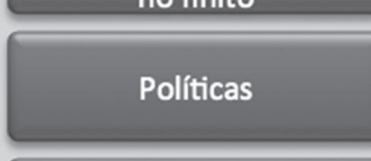

Partes interesadas

Gestión

Fuente. Elaboración propia de los autores, (s.f.). 


\section{REFERENCIAS BIBLIOGRÁFICAS}

Alba Hidalgo, D., Barbeitos Alcántara, R., Barral Silva, M., Benayas Del álamo, J., Blanco Heras, D., Domenech Antúnez, X., Fernández Sánchez, I., Florensa I Botines, A., García Orenes, F., López Álvarez, N., \& Ysern Comas, P. (2012). Sustainability and social responsibility strategies at Spanish Universities: An assessment tool. Profesorado, 16(2), pp. 59-75.

Alvarez Etxeberria, I. (2009). Sustainability reports as a tool to manage sustainability [Las memorias de sostenibilidad: Un instrumento para la gestión de la sostenibilidad]. Revista Española de Financiación y Contabilidad, 38(144), pp. 677-697.

Baker, S. (2005). Sustainable Development (Routledge Introductions to Environment: Environment and Society Texts). Routledge: New Ed edition, 1st edition.

Disterheft, A., Caeiro, S., Azeiteiro, U. M., and Filho, W. L. (2013). Sustainability Assessment Tools in Higher Education Institutions. In Caeiro, S., Filho, W. L., Jabbour, C., and Azeiteiro, U. M., editors, Sustainability Assessment Tools in Higher Education Institutions, pp. 3-27. Springer International Publishing, Cham.

Gallopín, G. (2003). Sostenibilidad y desarrollo Sostenible: un enfoque sistémico. Santiago de Chile: CEPAL.

Glavi, P. (2006). Sustainability engineering education. Clean Technologies and Environmental Policy, 8(1), pp. $24-30$.

Hanning, A., Abelsson, A. P., Lundqvist, U., and Svanstrm, M. (2012). Are we educating engineers for sustainability?: Comparison between obtained competences and Swedish industry's needs. International Journal of Sustainability in Higher Education, 13(3), pp. 305-320.

Hernández García, I. b., Hernández García, J. c., and Bernal, R. d. (2012). Alternative views of the city: Complexity, sustainability and everyday practices [Visiones alternas de ciudad: Complejidad, sostenibilidad y cotidianidad]. Bitacora Urbano Territorial, 20(1):67-77.

Huisingh, D. (2006). New challenges in education for sustainable development. Clean Technologies and Environmental Policy, 8(1), pp. 3-8.

Leff, E. (2000). Tiempo de sustentabilidad. Ambiente \& sociedad, (6-7), pp. 5-14.

Lozano, R. (2008). Envisioning sustainability three-dimensionally. Journal of Cleaner Production, 16(17), pp.18381846.

Martins, A. a., Mata, T. M., and Costa, C. a. V. (2006). Education for sustainability: challenges and trends. Clean Technologies and Environmental Policy, 8(1), pp. 31-37.

Mckeown, R., Hopkins, C. A., Rizzi, R., and Chrystallbridge, M. (2002). Manual de Educación para el Desarrollo Sostenible. Number 865. 
Mebratu, D. (1998). Sustainability and sustainable development: historical and conceptual review. Enviromental Impact Assesment Review, 18(6), pp. 493-520.

Méndez Chiriboga, M. A. (2012). La sostenibilidad y sustentabilidad en los museos, dos enfoques principales: La museología tradicional y la nueva museología. Estudio de caso en dos museos de la provincia de pichincha. Tesis previa a la obtención del título de licenciada en restauración y museología, Universidad Tecnológica Equinoccial.

Mulder, K. (2004). Engineering education in sustainable development: sustainability as a tool to open up the windows of engineering institutions. Business Strategy and the Environment, 13, pp. 275-285.

Mulder, K. F., Segalas, J., and Ferrer-Balas, D. (2012). How to educate engineers for/in sustainable development: Ten years of discussion, remaining challenges. International Journal of Sustainability in Higher Education, 13(3), pp. 211-218.

Niu, D., Jiang, D., and Li, F. (2010). Higher education for sustainable development in China. International Journal of Sustainability in Higher Education, 11(2), pp.153-162.

Pastor Seller, E. (2012). Sustainability, efficiency and impact of Social Policies and municipal social democratization it through involvement [Sostenibilidad, impacto y eficacia de las Políticas Sociales municipales mediante la democratización e implicación social]. Sociedade e Estado, 27(3), pp. 663-688.

Pujadas, C. (2011). Desarrollo sostenible o sustentable? ADN, (6), p. 28.

Saechsische, C.-G. (2013). Die Erfindung der Nachhaltigkeit. Leben, Werk und Wirkung des Hans Carl von Carlowitz. Oekom Verlag Munich.

Santos Rego, M. A. (2009). Migration, sustainability and education [Migraciones, sostenibilidad y educación]. Revista de Educacion, (SPEC. ISSUE), pp.123-145.

Tilbury, D. (2004). Rising to the challenge: Education for sustainability in Australia. Australian Journal of Environmental Education, 20(2), pp.103-114.

Vare, P. \& Scott, W. (2007). Learning for a Change: Exploring the Relationship Between Education and Sustainable Development. Journal of Education for Sustainable Development, 1(2), pp. 191-198.

Vilches, A. \& Pérez, D. (2012). La educación para la sostenibilidad en la Universidad: el reto de la formación del profesorado. Profesorado, 16(2), pp. 25-43.

Villamizar, F. (s.f.) ¿desarrollo sostenible? o ¿sustentable? (s.c) (s.f).

Viso, A. M. (2005). Sustainability and governance [Sostenibilidad y gobernanza]. Arbor, 181(715), pp. 317-331.

Waas, T., Hugé, J., Verbruggen, A., \& Wright, T. (2011). Sustainable Development: A Bird's Eye View. Sustainability, 3(12), pp. 1637-1661. 